\title{
Morphology of Tissue Disruption at Sites of High-Grade Tumors
}

\author{
Mousumi Chaudhury
}

\begin{abstract}
Background: Invasive solid cancers originating from diverse organs like breast, ovary and lung metastasize to distant sites. The structural changes at the primary sites of these high-grade tumors have not been well characterized. The main aim of the current study was to examine if there is any morphological overlap of metastasizing tissues of different invasive tumors.
\end{abstract}

Methods: Whole slide hematoxylin and eosin (H\&E) stained images from web repository of multiple tumor specimens were used for this study. ImageJ was used for image processing and analyses.

Results: The metastatic tissue(s) at the primary sites of different examined high-grade tumors appeared similar, irrespective of organ of origin of the primary tumor. Numerous excrescences with the repetitive appearance of a bulb-like projection with a narrowed-off trailing end were seen emanating from the tumor cell membrane. Many of them contained nuclei, while others were empty vesicles. Although these structures were not exactly equal in their dimensions, the rubrics of architectural distortion in different high-grade tumors were conserved.

Conclusions: The preliminary observations suggest for the first time that there is structural similarity of the epithelial dysmorphia in many highgrade invasive tumors, irrespective of their parental tissue of origin. This commonality of morphological prints of metastases suggests that similar pathways of cytosolic force generation are activated during temporal progression of cancer, resulting in the conserved mushroom-shaped appearance of the dismantling individual cell or cell clusters from the parental epithelium. The conserved genomic mechanisms underlying these fascinating observations merit testing and validation in future studies.

Keywords: Metastasis; Exosome; Cancer; Membrane; Circulating tumor cell; Dysplasia

\section{Introduction}

Metastasis in cancer is a critical cellular evolutionary phenom-

Manuscript submitted May 4, 2020, accepted July 2, 2020

Published online August 10, 2020

GIM Foundation, 1501 Rahling Road \#1006, Little Rock, AR 72223, USA. Email: chaudhurymousumi2015@gmail.com

doi: https://doi.org/10.14740/wjon1262 enon, which leads to the complexity of this disease. Spreading of cancer cells from its primary site to distant organs is a key factor that leads to worse prognosis for cancer patients. Although the tumor in the primary site could be managed with chemotherapy or surgical interventions, the dissemination of the cancer cells from its primary site is highly unpredictable, leading to guarded outcomes. Metastasis in cancer is combination of different processes. It involves lot of modification in cell morphology, signaling and genetic modification(s). The cancer cells which can successfully escape from the primary tumor and survive in the vascular tunnel, subsequently extravasate across the endothelium to seed into secondary sites. The secondary site also needs to be congenial and receptive to seed the primary tumor $[1,2]$. To survive its way to secondary sites, the disintegrating cellular mass needs to hide from immune cells, or they need to modulate the signaling process and rely on ligand-receptor interactions for the formation of specialized vesicular structures to facilitate this whole process of transition [3].

To acquire the characteristic of metastatic phenotype, the cancer cells need to evade its local tissue and adjacent blood and lymphatic vessels. The very first step to accomplish this property necessitates the cancer cells' requirement to migrate [4]. The migration of cancer cell requires high modification of its morphology and manipulation of the basement membrane where they are attached. The fundamental migratory property has been hypothesized to be achieved by formation of specialized vesicular structure [5]. Earlier, it was variously postulated that metastatic cancer were either non-healing wound [6] or over-healed wound [7]. However, the characteristics of these projections at the sites of human tumors have not been well characterized. In this present study, the morphology of these structures in different forms of aggressive human tumors from different organs of origin was examined. The observations reveal that the morphology of these structures in the membranes of high-grade tumors is almost same, irrespective of their organs of origin. Systematic study of these structures showed that these are highly conserved in their appearances and often spreading out as single cell or in clusters in lumen, disrupting from their primary epithelial sites. These structures occasionally contain some nuclear materials, while some of them are just empty membrane-bound vesicles.

Despite increasing evidence that there is some invasive structure like invadopodia which are conspicuous in metastatic tumor [8-11], and play a significant role in the invasion and metastasis of cancer, direct robust evidence of presence of these structures in situ in cancer slides and its real contribution 
towards pathology of disease in vivo is still lacking. The results of the present study show the repetitive appearance of ball and stick like excrescences in high-grade metastatic tumors, irrespective of the primary organ of origin, which likely suggests that these structures pull and separate out from the edge of the membrane and migrate to a distant site. The functional significance and step-wise pathophysiological processes resulting in this morphometric evolution at the origin of metastatic site still remain elusive, but likely suggests a commonality of mechanisms of cytoskeletal and membrane remodeling at the initiation of tissue disruption for dissemination to a different organ site.

\section{Materials and Methods}

\section{Web repository}

The present study was conducted using digitally archived images and no direct handling of human patients' sample occurred in the present study. Thus, no explicit institutional review board (IRB) approval was obtained. For above mentioned reasons, informed consent or ethical compliance with human/ animal study was not a requirement. The author considered it a privilege to be able to perform this current study due to the generosity of the slides made available by Prof. Rosai (https:// www.rosaicollection.org/).

Histopathological slides were examined from Dr. Juan Rosai surgical pathology seminar website (http://www.rosaicollection.org/), which has been graciously made freely available in the web by Prof. Rosai in collaboration with USCAP. Whole slide images from the web repository offered an opportunity for researcher for digital annotation, rapid navigation/magnification, and computer-assisted viewing and analysis. Whole slide images were used, with navigation of specific regions of interest (ROI) in the slide. Other important advantages in utilizing this website for the current analysis was the ability to annotate images, save ROI, take snapshots of selected regions, and export images to different formats. For detailed analysis, image analysis was used in conjunction with whole slide viewing tools. A popular free platform is ImageJ, a software tool made available by the National Institutes of Health (NIH) that includes several common image analysis algorithms useful for histology image processing. It is particularly important to find the desired types of tumor in limited time with key words from a huge repository. Knowledge of the case report behind each slide was also an important factor for choosing each tumor slide. Availability of numerous tumors slide of different organ of origin is key factor in choosing the website. Different web resources like The Cancer Genome Atlas (TCGA), Virtual Pathology at the University of Leeds, Web Pathology were also immensely helpful, but after careful consideration for the desired study purpose, "Juan Rosai Pathology" slides were chosen for this study.

Scanning of images can occur at multiple magnifications. Scanning at $\times 20$ magnification is usually acceptable for standard viewing and interpretation, including routine image analysis of hematoxylin and eosin (H\&E) stained slides. In order to reproduce the magnifications necessary for the present study of many diagnostic and research applications, the slides were viewed at sufficiently high resolution and with adequate color depth for study. Resolution is typically expressed by the websites in units of micron per pixel and color depth is expressed as the number of bits allocated to a pixel, which specifies the total number of distinct colors possible in the image.

\section{Rationality of choosing slides}

Cases have been studied based on the H\&E stained slides and on their diagnosis available for each case. Each organ or tissue-specific tumor was selected after systematically browsing all seminars. Control cases were chosen based on their diagnosis notes as noninvasive benign tumor conditions. Figures were prepared by using representative $\mathrm{H} \& \mathrm{E}$ stained of at least 50 cases of each diagnosed breast cancer, ovarian cancer, lung cancer, melanoma and sarcoma. Figures were used for analyses and prepared for representation based on clarity, relevance and quality of the image. Documented metastatic invasive tumor slides were chosen to examine the unique features of tissue disruption of high-grade tumors. Figures were presented using $\times 20$ magnification using Juan Rosai's site (https://www. rosaicollection.org/) features available for all cases.

\section{Using ImageJ for analyses of images}

ImageJ is a public domain Java image processing and analysis program made from NIH Image. It usually runs as an online application or as a downloadable application, on any computer with a Java1.5. It can edit, display, analyze, process, save and print 8-bit, 16-bit and 32-bit images. It can read many different image formats including TIFF, GIF, FITS, JPEG, BMP, and "raw image". It can support stacks and hyper stacks, a series of images that share one window. It is multithreaded program, so time-consuming operations such as image file reading can be performed in parallel with other operations. ImageJ can calculate area and pixel value statistics of user-desired selections. It can measure distances, margins, and angles. It can also create density histograms and line profile plot. It supports standard image processing functions such as contrast manipulation, sharpening, smoothing, edge detection and median filtering. It does geometric transformations such as scaling, flips and rotation. Image can be zoomed up to 32:1. All analysis and processing functions are available at any desirable magnification factor. The program supports any number of images simultaneously, limited only by available memory. Spatial calibration is also available to provide real world dimensional measurements in units such as millimeters (mm). Density or gray scale calibration is also available. ImageJ was designed with an open architecture that provides extensibility via Java plugins. ImageJ is being developed, plus the BBEdit editor and the Ant build tool. The author, Wayne Rasband (wsr@nih.gov), is a Special Volunteer at the National Institute of Mental Health, Bethesda, Maryland, USA. Detailed information for Linux, Mac OSX, MacOS9 and Windows installation of ImageJ is available at http://rsb.info.nih.gov/ij/docs/install/index.html. 
In this study, ImageJ was used for its different potentials. It was used to prepare the figures to show boundary. Images were chosen and opened with ImageJ and then IMAGE tab is selected. After that type, tab was chosen to covert the image in to 32-bit image. This 32-bit image was used to make pseudocoloring image to show the morphological features in the slide. For tracing the perimeter, this 32-bit image was opened in PROCESS tab and then FIND EDGES tab was chosen to trace the boundary. Then the contrast was adjusted to visualize the boundary clearly. To measure the perimeter length, ImageJ was used with same figure and ANALYZE tab was selected and MEASURE tab was chosen to find out the perimeter length in $\mu \mathrm{m}$. The data were plotted in Microsoft Excel to create a bar graph of perimeter measured for all cases of breast tumor. Pseudocoloring of images was performed for creating contrast and better visualization of membrane structures. Images were opened in ImageJ (NIH freeware) and converted to 16- or 32bit prior to choosing a pseudocolor, which is 16-color option from the LOOKUP TABLE (LUT) for creating efficient contrast. This was done to see the nucleus and boundary clearly in the figures.

\section{Statistics}

The $t$-test was use to compare between means. Cumulative data was expressed as mean \pm standard error of mean (SEM).

\section{Results}

\section{Distinctive cellular deformities are present in the boundary of the metastatic breast tumors}

The upper panels in Figure 1 are representative of benign breast conditions, and the lower panels are invasive breast cancers, obtained from the slide repository of Dr. Juan Rosai's pathological seminar collections. Note that H\&E stained slides of highly metastatic breast tumors have random projections of microvesicles throughout its cellular perimeter in contrast to micro-images of benign breast tissues.

All the upper panels represent control tissues showing benign conditions. In the upper panel, the first two figures represent two different cases of fibroadenoma. Well-organized cytoplasm and nucleus can be seen. Continuous cellular boundary and no apparent breakage are visible in the tissue. Most of the nuclei are similar in appearance. The color of the nuclei is almost same across the tissues for both cases, which is mainly due to their same phase of division. The third figure in the upper panel is benign breast fibromatosis. This also has uniform appearance across the tissue. No apparent breakage and projection from cellular boundary are seen. Nuclei are uniform and no randomness is visible. No cells are seen inside the lumen. Last figure in the upper panel represent Paget's disease of breast. Uniform boundary and no apparent excrescences can be seen. Cluster of cells are visible in the center of the lumen. They are detached from the cellular boundary. This floating mass of cells inside the lumen has organized cytoplasm and nucleus. No gross breakage in the boundary can be observed in the tissue. Uniformly stained nuclei can be seen throughout the tissue.

All the microscopic images in the lower panel represent invasive breast cancer. In the lower panel, the first figure on the left represents a case of intraductal carcinoma. Ill-defined cell border is clearly visible. Projection of vesicular structures is seen inside the lumen. Cells are seen in different phases of evolution. There are overall random tissue features that can be observed in both the cytoplasm and nucleus. Round to elongated nuclear shape and mild to moderate nuclear membrane irregularities are seen. Collar-like cluster of cells at the 12 o'clock position in the process of shredding can be noted. Cytoplasmic linkers can be seen surrounding the membrane. Some of these parts are broken. Cohesive cluster of cells are seen floating in the lumen. These floating cells have irregular margin. The shapes of these cells are different. Some of these cells have nuclei, while others do not. Cell margins are irregular, and shapes of the nuclei are different in the clusters. Some of the nuclei are pyknotic.

The second figure in the lower panel is another case of intraductal carcinoma. Ill-defined cellular boundary is consistent among the both cases. Cytoplasmic linkers can be seen broken abruptly throughout the boundary. Consistent cytomorphologic features including nuclear overlapping, low to intermediate nuclear to cytoplasmic ratios, round to elongated nuclear shape, finely vacuolated cytoplasm, mild to moderate nuclear membrane irregularities and syncytial clusters can be observed throughout the tissue. Spindle-shaped excrescents can be seen projecting out from the cellular periphery. These sprouting structures are present across the periphery. Some of these excrescents have apical nucleus like in the 3 o'clock position, while others do not have nucleus as observed in 6 o'clock position. Bare nucleus is also present in some parts as observed in 8 o'clock position. Dysplastic nucleus can be seen beneath the basement membrane as observed by dark stained nucleus in some region in H\&E stained slide. Some of these excrescents can be seen shredding or floating inside the lumen.

The third figure in the lower panel is a case of invasive lobular carcinoma. Chaotic cellular organization can be clearly visible in the microscopic slide. Cellular boundaries are wavy throughout. Repetitive morphological feature of tear drops like excrescences can be seen in the periphery. Most of these excrescences have nuclei, while others are empty without any nuclear material. Nuclei are present in the apical part of the projection. These excrescences are in different phases of their formation as observed in the slide. Some of them are shredding out from the boundary, while others are attached with membrane as observed in the 3 o'clock position. Nuclear overlapping, low to intermediate nuclear to cytoplasmic ratios and round to elongated nuclear shape can be seen throughout the periphery.

The last figure in the bottom panel is the same case of invasive lobular carcinoma from a different region. Wavy margin with tear drops like excrescences can be seen coming out towards lumen. Nuclei are also present in some excrescences attached with the basement membrane. Diverse morphological features are observed among the nuclei which are in the basement membrane. A line of shredding cells can be seen floating 

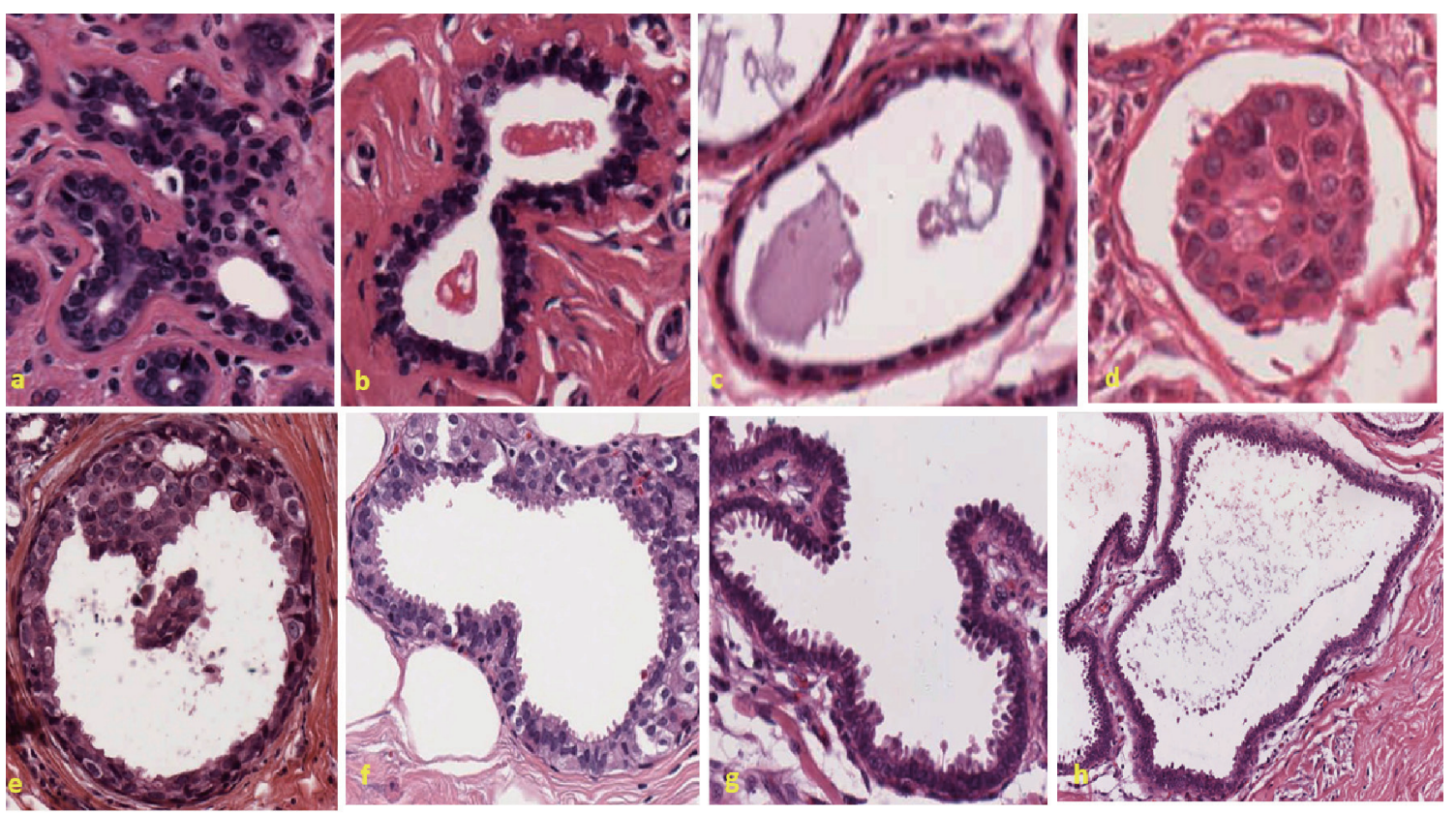

Figure 1. Pleomorphic changes in the cell membrane and egression of excrescents in benign and invasive breast tumors. Representative figure showing control tumors in the upper panel. Figure (a) represents a case of breast giant fibroadenoma. The slide shows well-circumscribed fibroepithelial growth, largely pericanalicular. Uniformly stained nucleus and cytoplasm are seen. The stroma is rather poor in cells and hyaline instead of myxomatous. Next figure (b) also represents another case of breast fibroadenoma. Well-organized cytoplasm and nucleus can be seen. Continuous cellular boundary and no apparent breakage are visible in the tissue. The stroma is fibrous and does not appear sarcomatous. Epithelial cells are not anaplastic. Figure (c) represents a case of breast fibromatosis. The cells are uniformly stained and no randomness in the arrangement of cytoplasm and nucleus is seen. The picture is one of relatively acellular fibrous tissue with a little fat and even less parenchyma, and no cancerous cell is observed in the slide. The last figure in the upper panel figure (d) represents Paget's disease of breast. There are no apparent dysmorphic changes in membrane and nuclear organization. Lower panel shows different cases of highly metastatic breast cancers. First figure in lower panel figure (e) shows intraductal carcinoma of breast. Highly random cell organization with different shapes and morphology of nuclei are seen. Cells seem like breaking from the basement membrane and some clusters of cells are seen floating inside the lumen. Next figure in the lower panel figure $(f)$ is a different case of intraductal carcinoma of breast. Asymmetric nucleus with formation of vesicular structure is seen unevenly throughout the margin. Breach in the basement membrane is clearly visible; also, some individual cells seem floating inside the lumen. Figure $(\mathrm{g})$ is a case of invasive lobular carcinoma breast. Convoluted membrane structure with lots of villi-shaped cells are projecting outward is clearly seen in this slide. The cells and especially the nuclei are considerably larger than normal or than those of benign proliferative lesion. Cell shapes are distorted and start of stromal infiltration is clearly visible. The case in the lower panel figure (h) is the same case of invasive lobular carcinoma but different region. Most of the features are same as those of previous slide. The cells are further distorted by the start of disintegration and stromal infiltration, and it seems like invasion appears to have progressed. Clusters of cells seem like shedding in pattern and going away from membrane.

inside the lumen. Some of them have nuclei, while others are anucleate or undergoing karyorrhexis.

\section{Cellular projections at metastatic site are similar in malig- nant breast tumors}

In Figure 2, the magnified image of invasive breast tumor unequivocally demonstrates bulbous and stalk like projections throughout the cellular perimeter. Images showing the tear drop like appearances are present among both cases of aggressive breast tumors. Both figures show excrescences arranged in beaded like appearance, attached to the cytoplasmic membrane. Nuclei present beneath the basement membrane are diverse in shape, color and morphology. Most of them are round to elongated with ill-defined cytoplasmic membrane and overlapping with each other, demonstrating the dysplastic appearance. The nuclei that are present in these spindle-shaped projections are apical in location. Although these structures are overall similar in appearance, the length and width of these structures are different for each excrescence, hinting at dynamic evolution of these structures.

\section{Ovarian tumors have similar spindle-like excrescences spreading throughout the cellular boundary}

In the upper panel, Figure 3 is representative of ovarian conditions which are benign, while lower panel represents invasive metastatic ovarian cancer.

First figure in the upper panel is shown to represent as control for ovarian tumor, which is benign case of ovarian the- 


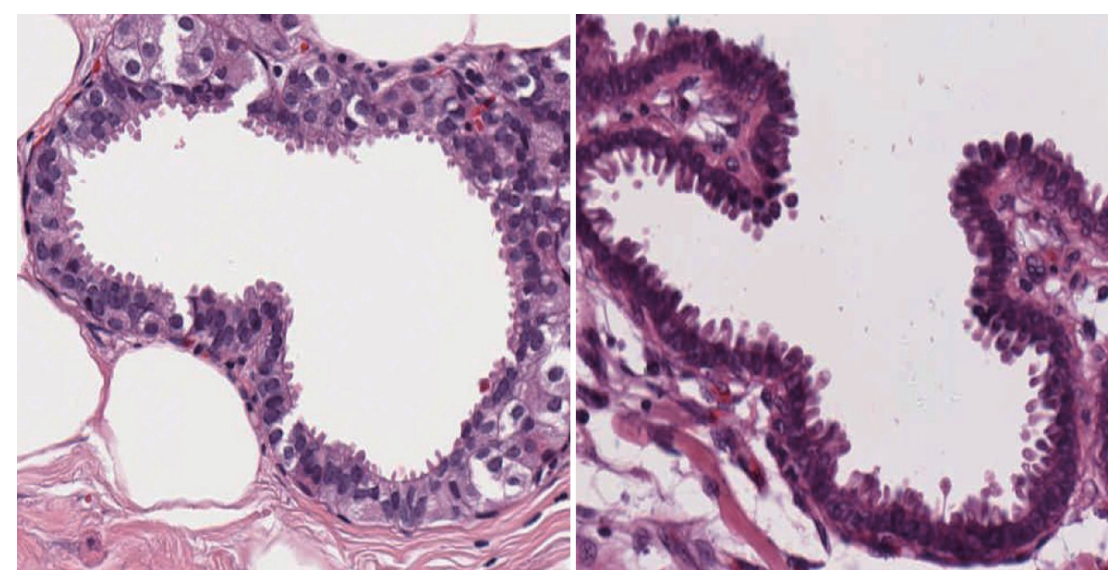

Figure 2. Random projection of vesicles surrounding the membrane. These figures show invasive intraductal carcinoma and invasive lobular carcinoma breast with lots of protrusion surrounding the boundary and with large number of excrescences or finger-like projections projecting towards the lumen randomly throughout the membrane.

coma. Well-organized cytoplasm and nucleus can be seen in the microscopic slide. Defined boundary with no breakage can be observed. Uniform boundary and no apparent excrescences can be seen. No floating mass or cells are present inside the lumen. Uniformly stained nucleus can be seen throughout the microscopic slide. Second figure in the upper panel is a case of polycystic ovary. This is also presented to represent as another control case in comparison with metastatic ovarian tumor. Well-defined boundary with no disruption in membrane is seen in the figure. Uniform cytoplasmic and nuclear feature is
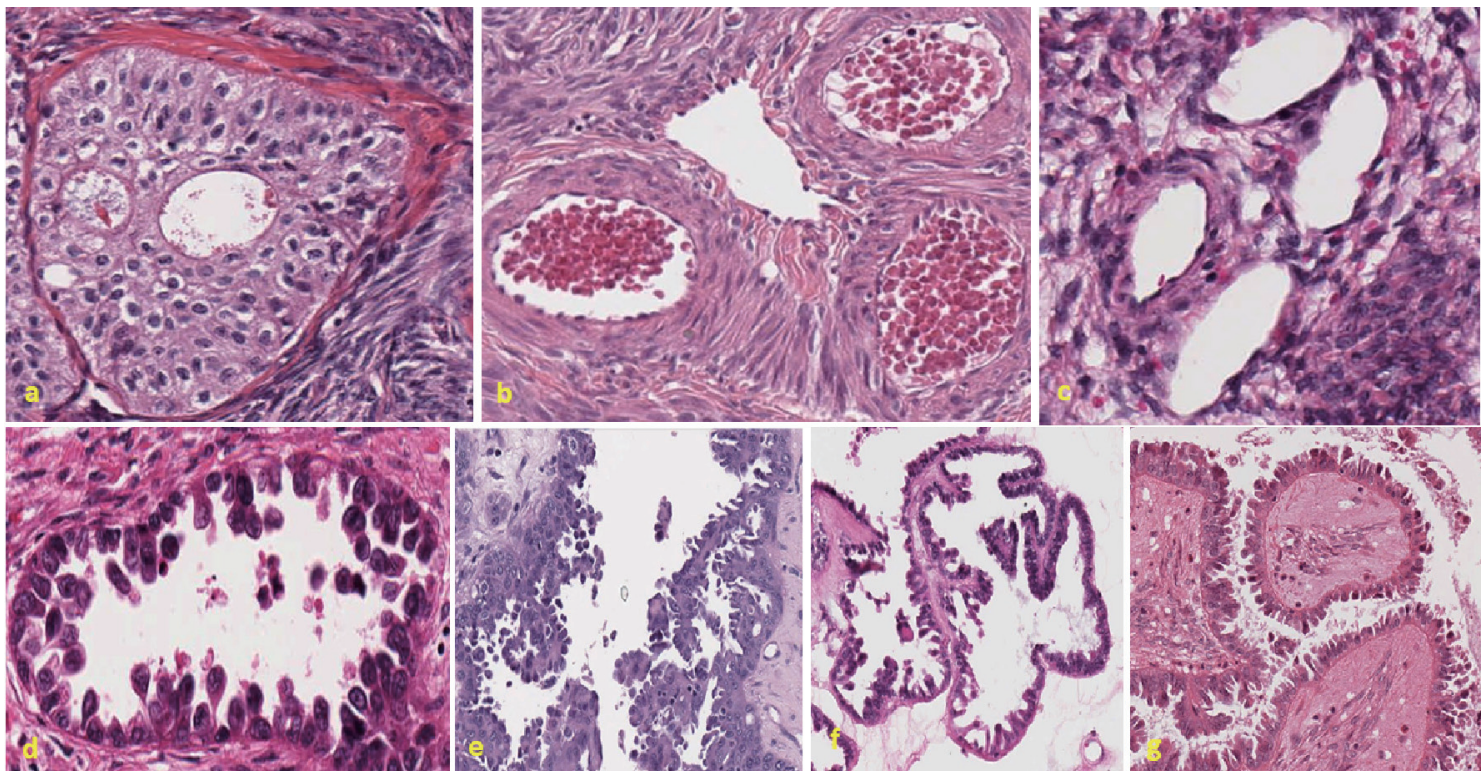

Figure 3. Ovarian tumors showing random projection of vesicles throughout the membrane. Representative figure in the upper panel showing control ovarian cases. The first figure in the upper panel figure (a) is a case of benign Brenner tumor of ovary. There is no apparent protrusion or degradation of the membrane. Cells are uniformly stained throughout the slide. Next figure (b) is a case of polycystic ovarian disease. Well-organized tissue slides with no malignant cells are visible in the slide. Last figure in the upper panel figure (c) represents a case of benign thecoma ovary. Evenly distributed cytoplasm and nucleus are seen, no membrane disruption is observed. Lower panel shows all metastatic cases of ovarian tumor. Figure (d) represents a case of endometrioid carcinoma ovary. Ball and stick-like structure are consistent throughout the slide and cells can be seen in different phase of their division. Cells seem like breaking and floating inside the lumen. Next figure (e) represents high-grade carcinoma ovary with diagnosis of serous, clear cell and endometrioid tumor. Highly random tissue organization and breakage of basement are clearly visible in this slide. Also, cells are floating in cluster or a single cell inside the lumen. Next figure (f) represents a case of serous carcinoma ovary. The arrangement of basement membrane is convoluted and villi-like cell projection can be observed throughout the slide. Last figure in the lower panel figure (g) also represents a case of high-grade serous carcinoma ovary. There is unevenly finger-like projection in the tumor membrane outside of the lumen. Random projections of vesicles surrounding the membrane in different structure and shape are seen. 

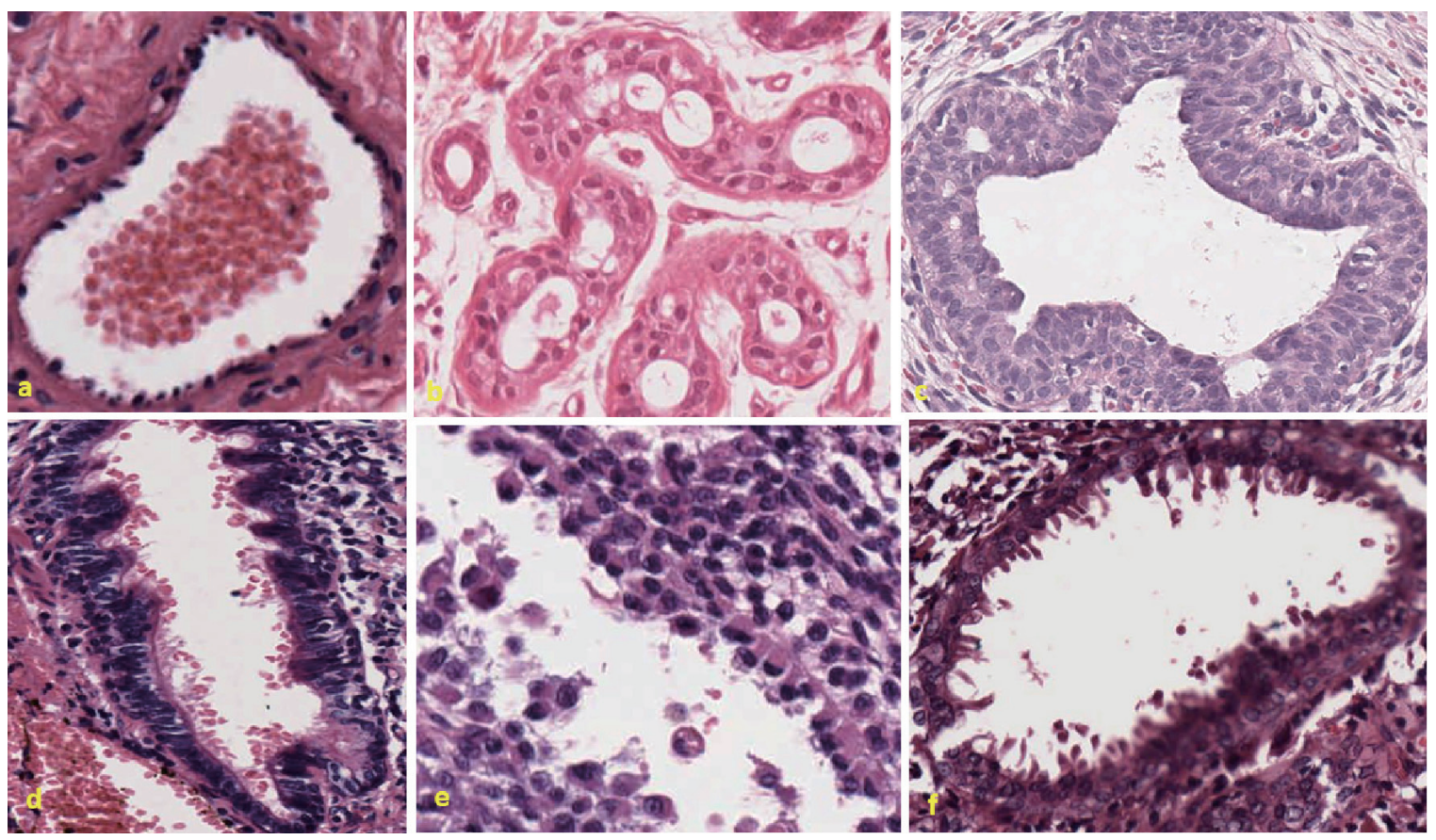

Figure 4. Different types of cancer have same morphological features of sprouting throughout the membrane. Figure (a) shows control lung hamartomatosis. Well-organized boundary throughout the slides is clearly visible. No randomness among the cells is observed. Next figure (b) represents a case of benign Bowen's disease of skin. Uniformly stained cells are observed all over the slide. No sign of breakage of basement membrane is observed. Next figure (c) represents a cellular giant fibroadenoma (benign cystosarcoma phylloides left breast) with organized cellular structure of cytoplasm and nucleus, respectively. In lower panel, the first figure (d) shows giant bone cell tumor, metastatic to the lung. Cells are highly disorganized and sprouting out from the membrane towards lumen. Cells are seen in different phase of division. Some individual cells are floating in the lumen. Next figure (e) shows metastatic malignant melanoma ovary. Highly disorganized cells with euchromatin and heterochromatin nuclei. Some cells have apical nuclei, while other do not. Cells are also breaking from the membrane and floating in clusters. Last figure (f) shows cystosarcoma phylloides of the breast. Highly pleomorphic nucleus and randomly scattered vesicular structures are visualized floating inside the lumen. This tumor has random and different phasic nuclei, irregularly dispersed cells with processes and vesicles are extending towards lumen.

also visible. Third figure in the upper panel is a benign case of Brenner's tumor. Uniform margins with well-organized cellular structure can be seen in microscopic slides. Nucleus looks similar throughout the tissue. No floating mass or shredding cells can be observed inside the lumen.

The first figure in the lower panel is a case of metastatic endometrioid tumor. Tear drops like excrescences are present across the cellular perimeter. Cytoplasmic linkers can be seen broken in some part of the surrounding membrane. Round to elongated nuclear shape and mild to moderate nuclear membrane irregularities are seen. The pattern of this microscopic feature is quite similar to metastatic breast tumors as shown in the lower panel of Figure 1. These excrescences are also seen floating inside the lumen either in single or in clusters. The nuclei that are present in the spindle are in different phases, both euchromatic and heterochromatic. Next three figures in the lower panels are different microscopic slides of high-grade serous carcinoma of ovary. Perimeters of these ovarian tumors are highly constricted at some part. Ill-defined cellular boundary is consistent among the all cases. Projections of vesicular structure are extensively seen inside the lumen. Tear drops like excrescences are uniformly seen in all cases. Apical nucleus is also common among all of them. Cohesive clusters of cells are seen floating inside the lumen. Some of these shredding excrescences are close to the basement membrane, while others are little away from the cellular boundary. Some of these excrescents are mostly intact in structure but others can be seen breaking at the portion as observed in third figure in the lower panel in the 6 o'clock position.

\section{High-grade tumors of diverse organs of origin have similar appearances throughout its cellular boundary}

In Figure 4, the upper panel is representative of benign conditions which has same organ of origin as corresponding to lower panel, which are invasive tumors.

Formation of finger-like projections in metastatic lung cancer, melanoma and cystosarcoma phylloides is seen. First figure in the upper panel is a case of lung hamartomata, a benign condition in the lung as control, in comparison with metastatic lung cancer in lower panel. Figure shows clear boundary, and no cells or metastatic mass are seen floating in the lumen. Cytoplasmic and nucleus organization are well defined. Second figure in upper panel is Bowen's disease of skin in early stage. Well-defined cellular boundary is seen. Uniformly or- 

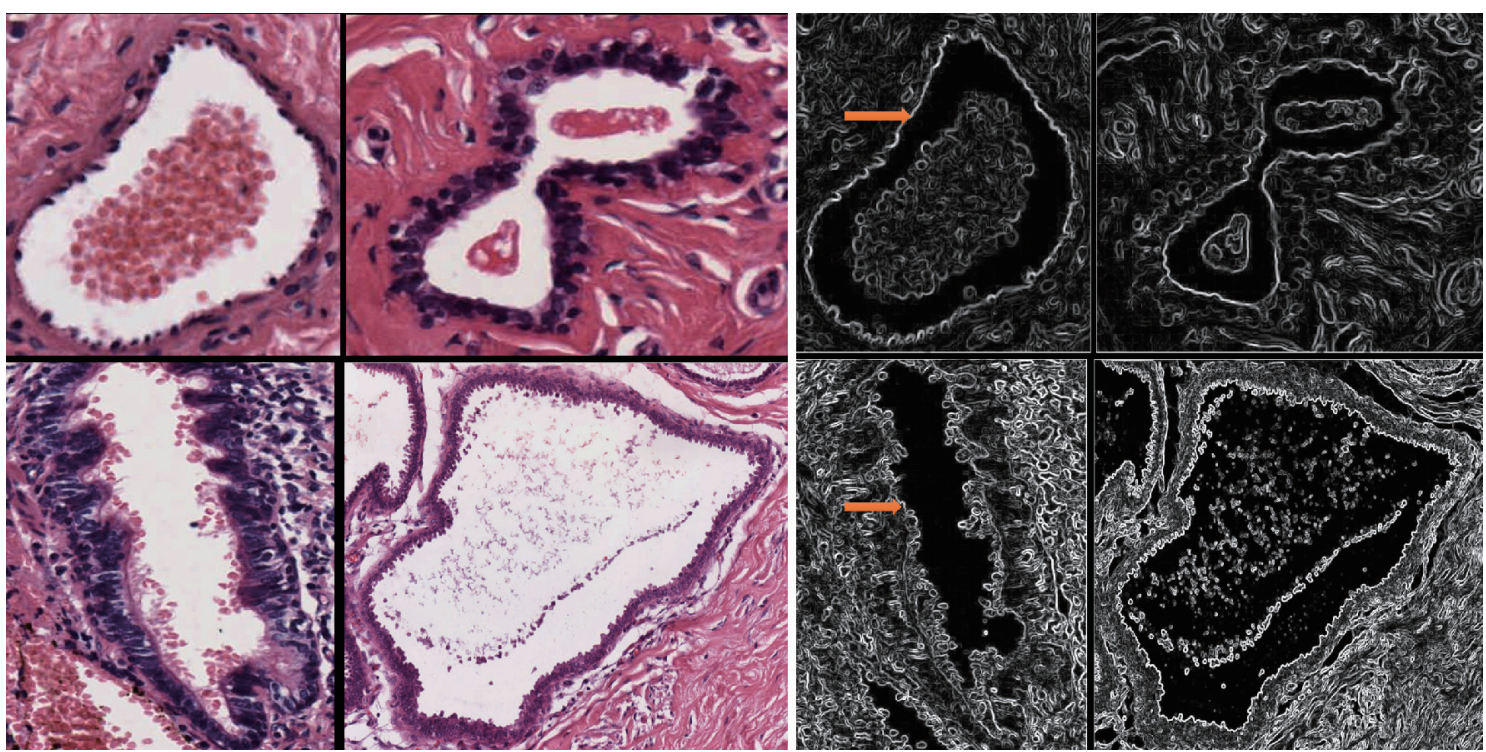

Figure 5. Metastatic tumors have convoluted and crooked margins than the control tumors, which have well-formed defined cellular margins. Representative figures in the upper panels showing controls. The first figure is showing control original H\&E staining of lung hamartomatosis, the next one is of fibroadenoma breast, and the next two images is of margins of the same control tumor represented with ImageJ perimeter measurement. White pseudocolored margins are well defined with no breakage or distortions throughout the perimeter. In the lower panel, the first two images show original H\&E staining of giant cell metastatic bone tumor to the lung, and invasive lobular carcinoma of the breast; the next two figures are the representation of margins with images of same tumors. Margins of metastatic tumors are highly crooked, distorted and not well defined. Orange arrows in the figures indicates waviness of the membranes of metastatic tumor in contrast with control tumor. H\&E: hematoxylin and eosin.

ganized cytoplasm and nucleus are seen. Third figure in the upper panel is a case of benign fibroadenoma. This also has defined boundary. No visible excrescences are seen. Uniformly present cytoplasm and nucleus are visualized. No shredding of cells can be seen in the lumen.

In the lower panel, the first figure is a case of giant cell metastatic bone tumor to the lung. Elongated excrescences can be seen throughout the perimeter of the cells delimiting the lumen. Cellular boundary is broken in some part of the cellular structure. Round to elongated nuclear shape, mild to moderate nuclear membrane irregularities and heavily pyknotic nuclei are seen. The appearance of this microscopic feature is quite similar as aggressive breast tumor explained in Figure 1, as well as high-grade ovarian tumor seen in Figure 3. Shredding excrescences can be seen floating inside the lumen. Second figure in the lower panel is metastatic malignant melanoma to the ovary. Microscopic image shows spindle-shaped excrescences throughout the boundary. Most of these excrescences has visible apically located nucleus. Some of the excrescences can be seen at the 12 o'clock position, almost at the verge of breaking free from the basement tissue. Nuclear overlapping, low to intermediate nuclear to cytoplasmic ratios, and round to elongated nuclear shape are seen. Cohesive cluster of cells or individual excrescences are seen floating in the lumen. Some of these cells have nuclei, while others do not. Also, the euchromatic and heterochromatic nucleus can be seen floating in the lumen. Last figure on the right in the lower panel is microscopic image of malignant case of cystosarcoma phylloides breast. Wavy margin with tear drops like excrescences can be seen coming out towards the lumen. Nuclei are also present in some excrescences attached with the basement membrane. Highly pleomorphic nuclei and randomly scattered vesicular structures are seen floating inside the lumen. Tear drop like excrescences can be seen throughout the periphery. Most of them are attached with the basement membrane. Shredding cells are seen floating inside the lumen.

\section{Wavy, serrated margin and increase in cellular perimeter in highly metastatic tumor}

The metastatic tumors are characterized by their wavy marginal pattern as seen in Figure 5. The first two figures in upper panel with H\&E stained showing control cases of lung hamartomatosis and the next one is of fibroadenoma breast. The next two pseudocolored images is of margins of the same control tumors represented with ImageJ perimeter marking application for the purpose of clear visualization. The first two figures in the lower panel show original H\&E staining of metastatic giant cell tumor of bone to lung, and invasive lobular carcinoma of breast. The next one is the representation of margins with images of the same tumor. Wavy and serrated margins are distinctive in metastatic tumors in contrast with control tumor. Orange arrow in upper panel shows the less wavy pattern part of cellular perimeter, while orange arrow in the lower panel shows highly serrated margin. Pseudoimaging of black and white color shows shredded excrescences are coming out from the cellular perimeter. Shredding excrescences can also be seen floating inside the lumen in the lower panel last image, as observed like white dots in the lumen. 
Increase in Cellular Perimeter in Metastatic Breast Tumors

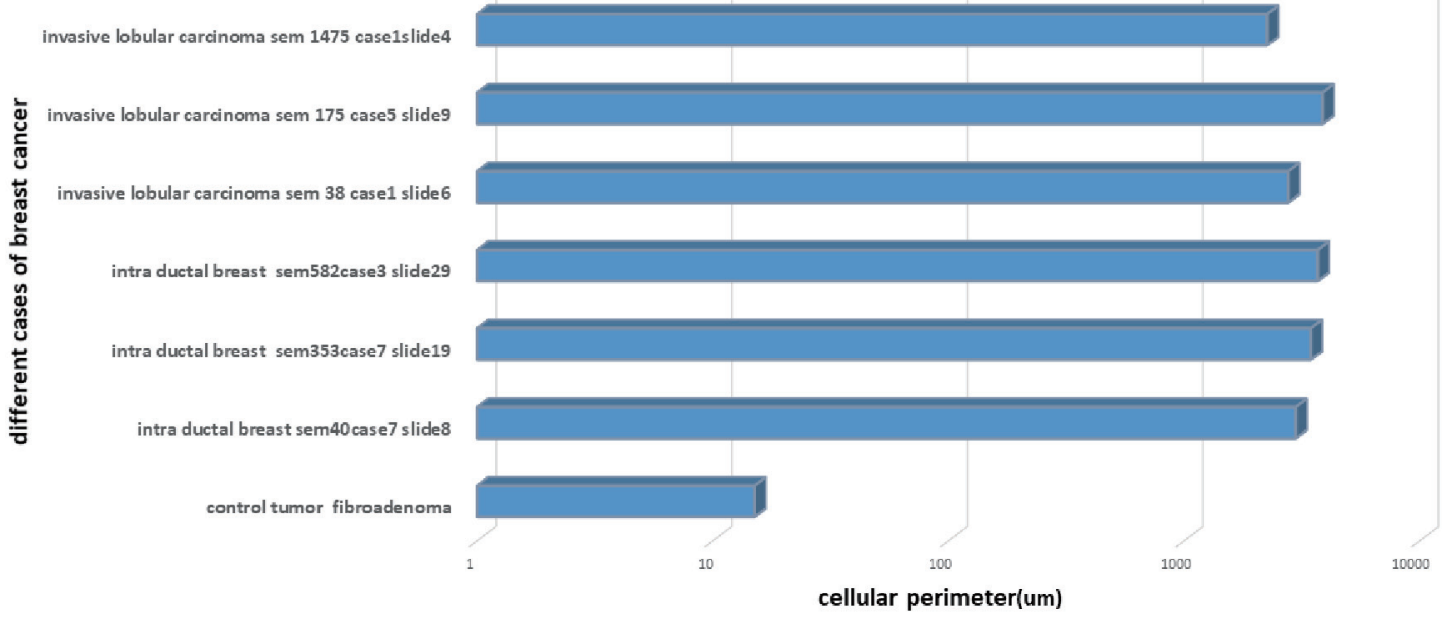

Figure 6. Quantitative measurement of increase in membrane perimeter in invasive cancer. There is distinctive increase in membrane perimeter in invasive breast tumor measured with the Image J tools compared with benign breast case. Increase in perimeter is shown in blue bar graph with $\mu \mathrm{m}$ unit. There is nearly 100 -fold increase in length of perimeter in invasive breast tumor.

The bar graph in the Figure 6 shows significant increase in the cellular perimeter in case of highly metastatic breast tumor, as compared to control breast fibroadenoma. ImageJ software was used to measure the perimeter of all different cases of invasive breast cancers and control breast conditions. Images were first chosen and converted to 32-bit image. This was measured with ImageJ analyze tools. These measurements were then plotted in Microsoft Excel. Increase in cellular perimeter was about 100-fold higher as observed in the Figure 6, in comparison to control perimeter as observed in the blue bar $(2,712.86 \pm 496.51,15.0 \mu \mathrm{m}$ vs. $3,162.84 \mu \mathrm{m}, \mathrm{P}=0.0016, t$ test, GraphPad QuickCalcs). This may be a possible indication of membrane synthesis in metastatic tumors.

\section{Breakage in the basement membrane and presence of bare nucleus}

Figure 7 shows the microscopic image of invasive intraductal carcinoma breast. Figure in the left is the original image of tumor slide with $\times 20$ magnification. Figure on the right side is the pseudocolored image of the same slide transformed using ImageJ for enhanced demonstration purpose. There is visible breakage in the basement, which can be observed

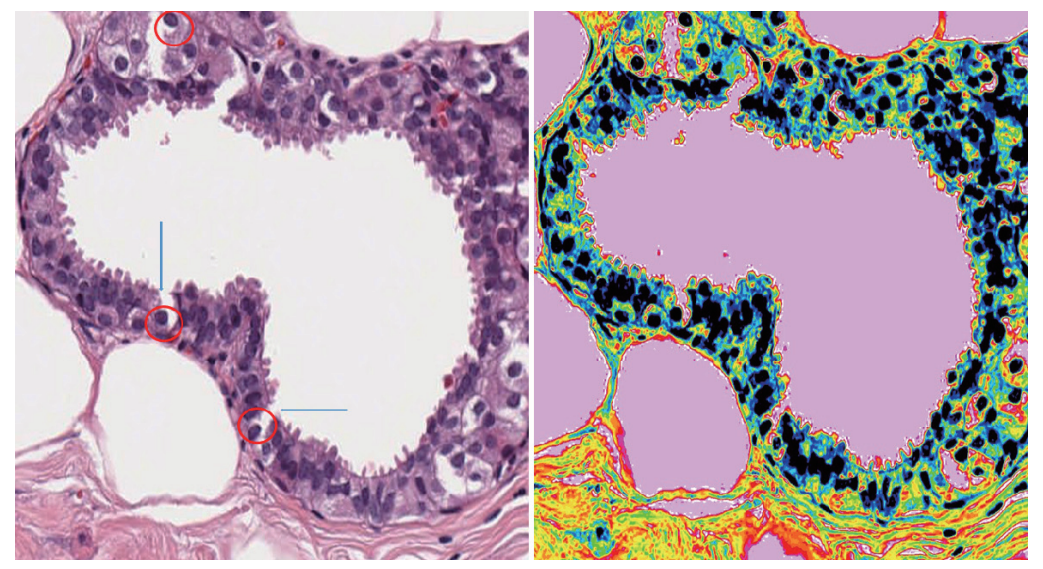

Figure 7. Breaching of basement membrane is more apparent in the tumor where there are lot of excrescences sprouting along the membrane in invasive breast cancer. This figure shows the microscopic image of invasive intraductal carcinoma breast. Figure on the left is the original image of tumor slide with $\times 20$ magnification. Figure on the right-hand side is the pseudocolored image of the same slide made using ImageJ for clear demonstration purpose. This has random breakage of basement membrane. There are numerous dysplastic cellular arrangements through the lumen and marked nuclear polymorphism. Breakage of basement membrane is found also uncovering the nucleus, leaving it naked (represented with red circle) and with blue arrow. There are some floating vesicles seen in the lumen. The next panel shows pseudocolored image of the same slide, where floating vesicles is clearly visible. 


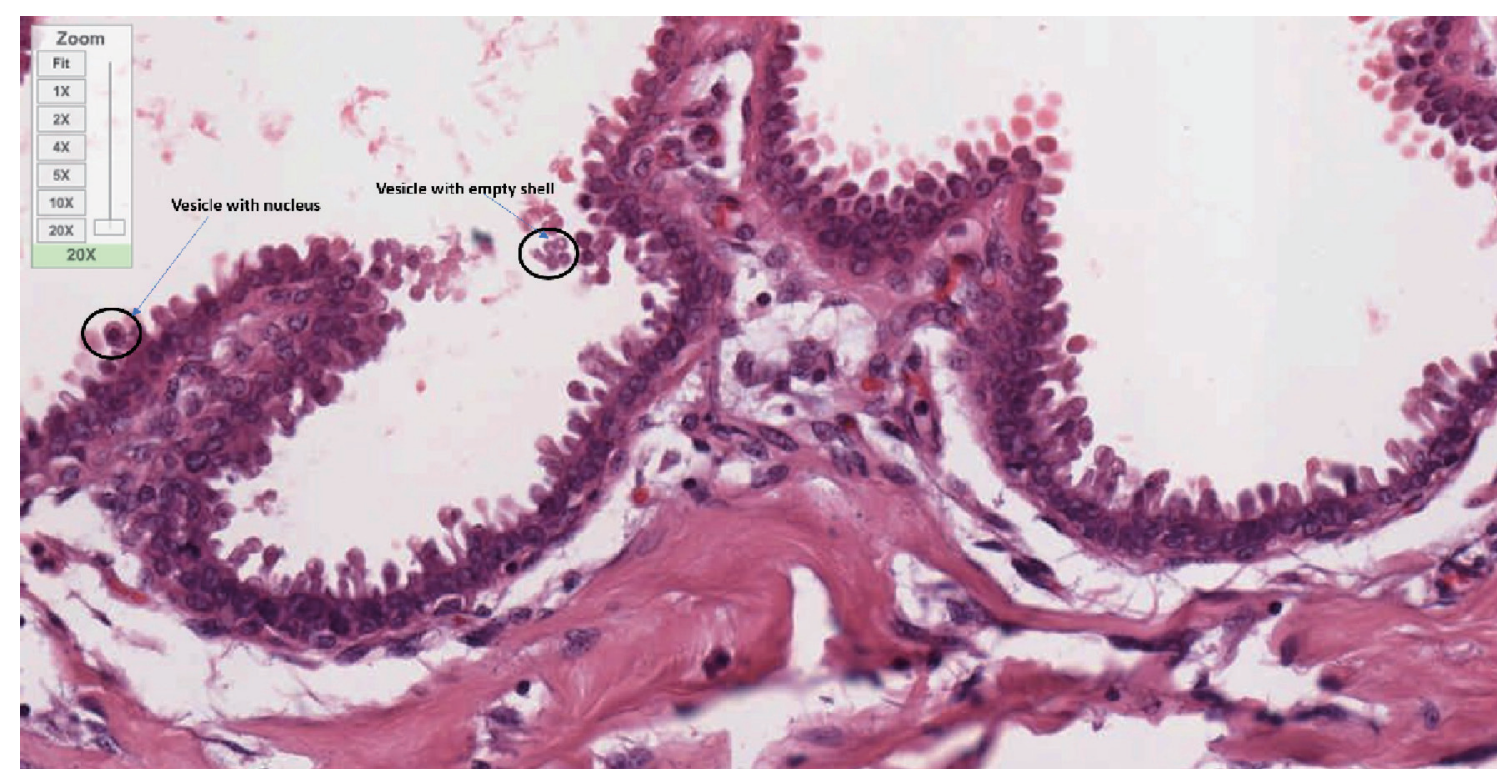

Figure 8. Distinct mushroom-like projections with oval head and cylindrical stalk coming out from membrane. This figure is a $\times$ 20 magnified image of invasive lobular carcinoma of breast. Finger-like vesicular projections are seen coming out from the membrane. All these vesicles are different in length but have almost uniform morphology. Some of them are attached with membrane and others are detaching from the membrane and migrating towards the lumen in cluster. Some of these vesicles are just empty and some of them carrying nucleus as marked with black circle and blue arrow.

throughout the cytoplasmic linker. This breaking has no pattern and mostly a little distance apart from previous breakage. Projection of excrescences can be seen spreading across the basement membrane. Bare nucleus can be seen in the path of breakage of basement membrane. Vacuolated cells with large nucleus can be seen in the figure. Binucleated or multinucleated nucleus with high nuclear cytoplasmic ratio is also present in the figure. In the right side of the pseudocolored image, the projection of cells is more apparent with orange color bordering. Pseudocolored image shows that the borders are mostly continuous even in the broken parts of basement membrane. This indicates the possibility of invagination or disruption. The excrescences that are already completely detached have their own boundary, possibly indicating membrane synthesis.

\section{Distinctive morphological features of tear drop shaped ex- crescents in invasive breast cancer}

This Figure 8 is the $\times 20$ magnified image of invasive lobular carcinoma breast. The projection of villi-like excrescent can be seen through the basement membrane. Overall morphology of these projections is similar with oval head and stick-like thin filament supporting the head. There is difference in the length and width of this feature. This could be due to their different phase of formation. Most of these excrescences are standing alone while some are overlapping each other. Most of these excrescents have nuclei in their head while some are just empty vesicles, which are visible as white center and pointed out in the figure. Some of these excrescences started shredding in a cluster observed at 11 o'clock position, while some are shredding as individual entity as seen in 3 o'clock position. Nuclei of different shapes, size and at different phases of development are visible in different layers of the basement membrane. Overall chaotic architectural status is visible in this tissue image.

\section{Morphological commonality of excrescences is present ir- respective of the tumor of origin}

Figure 9 represents the magnified image of excrescences observed in different type tumor tissues. Slides are chosen from each case based on the clear visibility of this unique feature of excrescent budding out from the cellular boundary. Upper panel is showing all magnified original images of excrescents, while lower panel is showing pseudocolored image of same tissue, processed with ImageJ. Sixteen-color options of corresponding image are shown. First figure is of invasive lobular carcinoma breast. Feature shows uniform tear drop like structure, with the apical part filled with nuclear cytoplasmic material as shown in light blue and dark blue in the middle of the pseudocolored image. This whole structure is surrounded with continuous line of membrane as shown in orange. Below this projection lies layer of nucleus as observed in dark blue. Next panel is also from the same case of invasive lobular carcinoma. This image shows apical nucleus inside the projection sprouting out from below. Some of these excrescences have single nuclei. One excrescence can be seen having binucleated and in the process of separation from each other. The next panel is also from invasive lobular carcinoma showing presence of excrescent without any nuclear material as seen in yellow color inside the tear drop shaped structure. Also, different intensity 

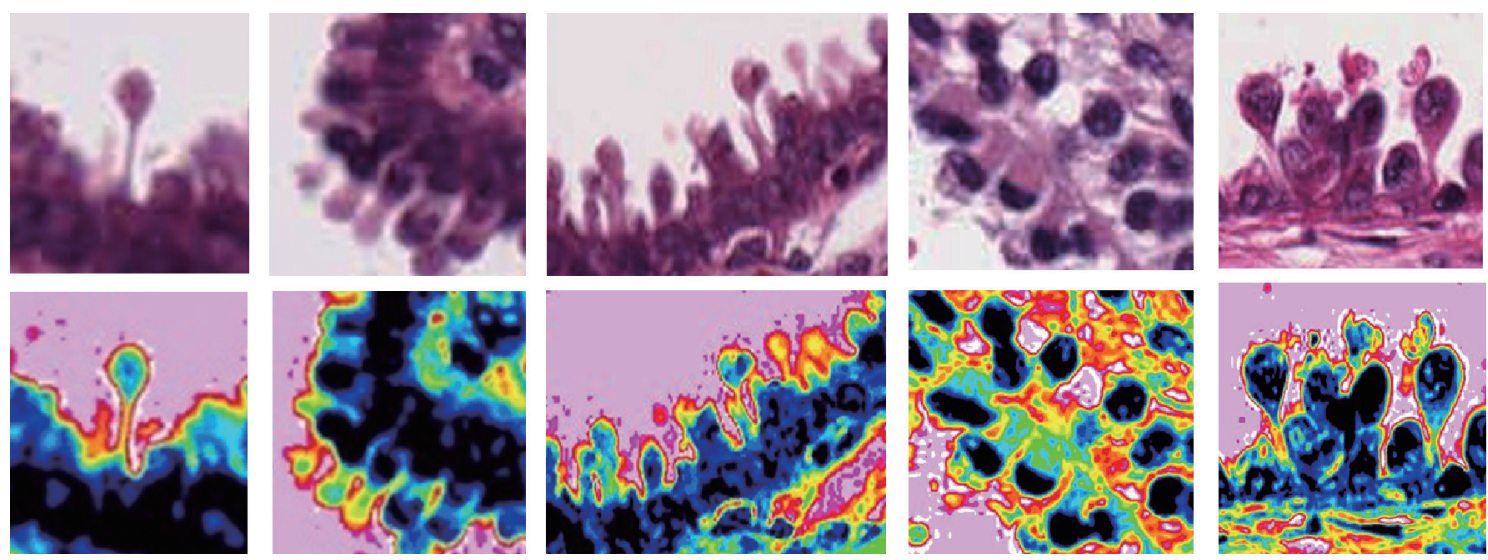

Figure 9. Different shapes and morphological structure of budding excrescents from different invasive cancers (First three panels, invasive lobular carcinoma of breast; malignant melanoma; ovarian endometrioid tumor). This figure shows different forms of budding vesicles in invasive cancers. Upper panel shows all magnified original image of excrescents, while lower panel shows pseudocolored image of the same tissue done with ImageJ 16-color options corresponding image with upper panel. All these projections have common rounded or oval-shaped head with cylindrical stalk. Some of them are well formed, while others are in the process of formation. Few of these structures are with nuclei, while some are without nuclei. The morphology of these projections is quite random throughout the perimeter.

of dark blue coloration beneath the membrane is indication of cells in their different phase of life cycle. Fourth panel is depicting the morphology of excrescents from metastatic malignant melanoma. Here also this projection has clear boundary and apical nucleus in the head. Binucleated nucleus can also be seen inside the vesicles. Last panel is a case of ovarian metastatic endometrioid tumor. Tear drop shaped appearance of excrescent is clearly visible in the pseudocolored image. Dark blue nuclei are seen in most excrescents. Some nuclei are in euchromatic stage, while others are in heterochromatic phase. Some of these excrescents are also attached with each other. Few of the excrescents also seems not in proper tear drop like appearance as in 1o'clock position. Different variations in the size of projections are clear indication of different phases of formation or degradation of these excrescents. Overall, this membrane-delimited extrusion structure is similar in different types of malignant tumors.

\section{Discussion}

The results of the current study show that: 1) Highly metastatic breast tumors have random projections of membrane blebs throughout its cellular perimeter in contrast to benign breast conditions. These structures show repetitive symmetry, resembling a racket or in the form of a ball and a stick. 2) Ovarian tumors have similar bulb and stalk structures just like invasive breast tumors sprawling around the cellular perimeter, whereas absent in normal ovarian conditions. 3) Formation of fingerlike projection is also present in other high-grade tumors arising from diverse organs, like metastatic lung, melanoma and cystosarcoma phylloides tumors, in contrast which are absent in their corresponding tissues under benign proliferative conditions. The results of the present study show the intriguing observation that in many metastatic tumors examined, there is formation of villi-like or finger-like excrescences, which may vary in their size, but have overall same features irrespective of their tissues of origin.

Though preliminary in scope, the observations of the present study have important implications. That the exosomal structures of high-grade tumors have repetitive morphology indicates similarity in morphogenesis of exosome and microvesicles, irrespective of the primary organ of origin. It is likely that mechanical force has a major contribution in this aspect, leading to the formation of these symmetric finger-like projections all over the membrane $[12,13]$. The differential locations of the nuclei in these excrescences also support the inference of generation of stochastic and chaotic cellular forces and their action in the biogenesis of these membrane-bound extensions, which likely are the first step(s) in generation of distant organ metastasis [11]. Different types of the curvature-favoring proteins like the Bin/amphiphysin/Rvs (BAR) domain-containing proteins were shown to drive the formation of mushroom-like structure tubular and vesicular membrane structures [14-16]. The upregulation of function of these classes of proteins merits examination in future studies.

In any system the ability of cells to migrate and invade through the surrounding extracellular matrix and across tissue boundaries are crucial [17]. This migration is one of the fundamental processes not only for physiological aspects that occur during normal development in embryonic phase, wound healing, and immune responses, but also important for the progression of disease conditions such as arthritis, vascular diseases and cell invasion and metastasis [18, 19]. One of the major steps in migration is cytoskeleton remodeling, reorganizing and reforming their actin cortical cytoskeleton to facilitate this movement. Many tumors in progression are characterized by the spread of the cells from the primary site of origin to the distant site. Extensive studies have been performed to understand the mechanism of this distant spread. However, there has been only scant examination on the morphology of these metastatic structures. It has been suggested 
that for epithelial tumors, the cellular phenotype undergoes transition and acquires the ability for motility by transformation into a mesenchymal variety and acquisition of motor proteins [20]. Humoral factors like transforming growth factor (TGF)-beta have been implicated in these epithelial to mesenchymal transition [21, 22]. Many other high-grade tumors produce large extracellular vesicles. They include pancreatic ductal adenocarcinoma, cholangiocarcinoma, hepatocellular carcinoma, colorectal carcinoma, glioblastoma multiforme and gynecologic malignancies (preliminary observations, unpublished). Previous studies have shown that high-grade tumors dedifferentiate to a more primitive phenotype [23]. This is likely a fundamental event ensuing with local uncontrolled overgrowth and universal phenomenon irrespective of the organ of origin of a tumor. The takot-tsubo-like membrane excrescences may play a seminal role in containing the nuclei for genomic dissemination to a distant site. Whether the tail-end of this excrescenting structure plays a role in intravascular travel of the circulating tumor cell (CTC) is currently unknown. Coordination of cytosolic forces is needed for nuclear egression and packaging in a membrane-bound structure for distant dissemination. Enucleation in erythrocytes or mammalian lens epithelia or keratinocytes may provide insights into such processes at sites of high-grade dysplasia, carcinoma in situ and high-grade invasive cancers [24]. A goal of the laboratory remains to study the coordinated action of the force-generating motor proteins and its genomic regulation in genesis of these membrane-bound excrescences. These studies may aid in development of targeted cancer therapy.

\section{Acknowledgments}

The author expresses deep gratitude to Dr. Juan Rosai. This article is dedicated to my mothers' Mrs. Tapasi Chaudhury and Mrs. Madhu Chhanda Chatterjee.

\section{Financial Disclosure}

None to declare.

\section{Conflict of Interest}

None to declare.

\section{Informed Consent}

Not applicable.

\section{Author Contributions}

MC has conceptualized, studied, analyzed and drafted the manuscript.

\section{Data Availability}

The data supporting the findings of this study are available from the corresponding author upon reasonable request.

\section{References}

1. Paget S. The distribution of secondary growths in cancer of the breast. 1889. Cancer Metastasis Rev. 1989;8(2):98101.

2. Chambers AF, Groom AC, MacDonald IC. Dissemination and growth of cancer cells in metastatic sites. Nat Rev Cancer. 2002;2(8):563-572.

3. Carman CV, Springer TA. Trans-cellular migration: cell-cell contacts get intimate. Curr Opin Cell Biol. 2008;20(5):533-540.

4. Petrie RJ, Yamada KM. At the leading edge of threedimensional cell migration. J Cell Sci. 2012;125(Pt 24):5917-5926

5. Sedgwick AE, D'Souza-Schorey C. Extracellular microvesicles and invadopodia mediate nonoverlapping modes of tumor cell invasion. Sci Rep. 2015;5:14748.

6. Dvorak HF. Tumors: wounds that do not heal. Similarities between tumor stroma generation and wound healing. N Engl J Med. 1986;315(26):1650-1659.

7. Schafer M, Werner S. Cancer as an overhealing wound: an old hypothesis revisited. Nat Rev Mol Cell Biol. 2008;9(8):628-638.

8. Murphy DA, Courtneidge SA. The 'ins' and 'outs' of podosomes and invadopodia: characteristics, formation and function. Nat Rev Mol Cell Biol. 2011;12(7):413426.

9. Eckert MA, Yang J. Targeting invadopodia to block breast cancer metastasis. Oncotarget. 2011;2(7):562-568.

10. Buccione R, Caldieri G, Ayala I. Invadopodia: specialized tumor cell structures for the focal degradation of the extracellular matrix. Cancer Metastasis Rev. 2009;28(12):137-149.

11. Yilmaz M, Christofori G. EMT, the cytoskeleton, and cancer cell invasion. Cancer Metastasis Rev. 2009;28(12):15-33.

12. Jayasinghe C, Simiantonaki N, Kirkpatrick CJ. Histopathological features predict metastatic potential in locally advanced colon carcinomas. BMC Cancer. 2015;15:14.

13. Wirtz D, Konstantopoulos K, Searson PC. The physics of cancer: the role of physical interactions and mechanical forces in metastasis. Nat Rev Cancer. 2011;11(7):512522.

14. Torzilli PA, Bourne JW, Cigler T, Vincent CT. A new paradigm for mechanobiological mechanisms in tumor metastasis. Semin Cancer Biol. 2012;22(5-6):385-395.

15. Khatau SB, Bloom RJ, Bajpai S, Razafsky D, Zang S, Giri A, Wu PH, et al. The distinct roles of the nucleus and nucleus-cytoskeleton connections in three-dimensional cell migration. Sci Rep. 2012;2:488.

16. Arkhipov A, Yin Y, Schulten K. Four-scale description of membrane sculpting by BAR domains. Biophys J. 
2008;95(6):2806-2821.

17. Makale M. Cellular mechanobiology and cancer metastasis. Birth Defects Res C Embryo Today. 2007;81(4):329343.

18. McNeil PL, Ito S. Molecular traffic through plasma membrane disruptions of cells in vivo. J Cell Sci. 1990;96(Pt 3):549-556.

19. Revach OY, Weiner A, Rechav K, Sabanay I, Livne A, Geiger B. Mechanical interplay between invadopodia and the nucleus in cultured cancer cells. Sci Rep. 2015;5:9466.

20. Lambrechts A, Van Troys M, Ampe C. The actin cytoskeleton in normal and pathological cell motility. Int J Bio- chem Cell Biol. 2004;36(10):1890-1909.

21. Chaudhury A, Howe PH. The tale of transforming growth factor-beta (TGFbeta) signaling: a soigne enigma. IUBMB Life. 2009;61(10):929-939.

22. Kalluri R. The biology and function of exosomes in cancer. J Clin Invest. 2016;126(4):1208-1215.

23. Wu PH, Phillip JM, Khatau SB, Chen WC, Stirman J, Rosseel S, Tschudi K, et al. Evolution of cellular morphophenotypes in cancer metastasis. Sci Rep. 2015;5:18437.

24. Ji P, Murata-Hori M, Lodish HF. Formation of mammalian erythrocytes: chromatin condensation and enucleation. Trends Cell Biol. 2011;21(7):409-415. 